\title{
Une centrale hydroélectrique clé en main, réversible et tête de vallée à Thissavros (Grèce)
}

\author{
T. Benassis et R. Pascal \\ Département Hydraulique de Cegelec
}

\section{T THISSAVROS, UNE CENTRALE CLÉ EN MAIN}

Suite à une compétition internationale et avec imposition de part locale importante, en particulier en ce qui concerne la fabrication des turbines et des alternateurs, PPC, Public Power Corporation, l'équivalent d'EDF en Grèce, a confié le 13 mai 1991, le contrat « clé en main » pour la fourniture des équipements électromécaniques de la centrale hydroélectrique turbine-pompe de Thissavros au Groupement piloté techniquement par Cegelec et formé des Sociétés Cegelec (France) - Metka (Grèce) - Alsthom Jeumont (France) - Hydro Vevey (Suisse) (fig. 1).

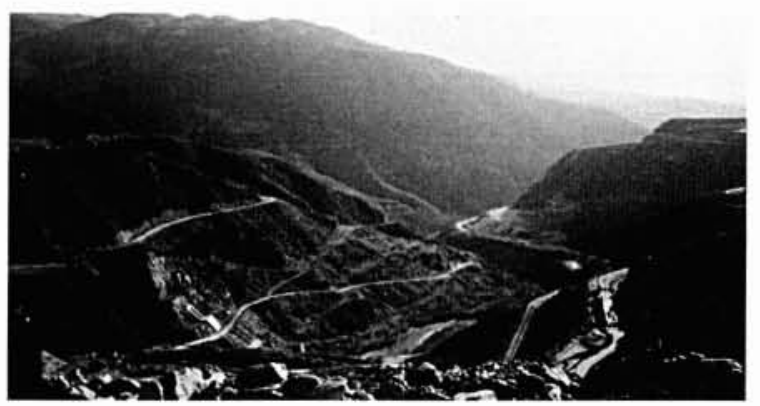

1. Photo générale du site de Thissavros:

Rappelons que Cegelec, la Branche Ingénierie du groupe Alcatel Alsthom, a acquis dans le domaine des centrales hydroélectriques, une large expérience en tant que coordonnateur de groupement et est à même de proposer des solutions cohérentes pour tout type de projet en particulier pour ceux de centrales turbine-pompe.

En effet, Cegelec a coordonné les plus importantes centrales de turbinage-pompage récemment construites: Guangzhou $(4 \times 300 \mathrm{MVA})$ en Chine, Muju $(2 \times 300 \mathrm{MVA})$ en Corée.

Pour ce type de contrat, Cegelec exerce notamment les rôles de coordonnateur de consortium, d'entrepreneur électricien et électro-mécanicien, et celui de fournisseur de systèmes de contrôle-commande.

Dans le Groupement en charge de la réalisation de Thissavros, les fournitures et prestations se répartissent comme suit :
- Alsthom Jeumont (France) fournit les moteursalternateurs,

- Hydro Vevey (Suisse) les turbines et les vannes sphériques,

- Metka (Grèce) le poste 150/20 kV, les transformateurs, les câbles $150 \mathrm{kV}$, la distribution basse tension, les auxiliaires mécaniques, et est partenaire d'Alsthom Jeumont et d'Hydro Vevey pour leurs fabrications,

- Cegelec (France) fournit les câbles (hormis $150 \mathrm{kV}$ ), les jeux de barres et le contrôle-commande.

Cegelec (France) est pilote technique du Groupement ; à ce titre, il conçoit l'architecture générale, implante les matériels des différents fournisseurs, réalise les plansguide de génie civil et les schémas de coordination de la partie électromécanique.

A toutes les étapes, depuis les études jusqu'à la mise en service, Cegelec établit et actualise périodiquement le planning général de l'affaire, organise et dirige les réunions consortiales. Enfin, il coordonne les travaux de montage des éléments électriques et électromécaniques.

II THISSAVROS, UNE CENTRALE TURBINE POMPE

\subsection{L'aménagement du fleuve Nestos}

Le cours d'eau bulgare Mesta devient Nestos en traversant la frontière grecque, puis serpente dans les montagnes de la Macédoine pour se jeter dans la mer de Thrace, près du port de Kavala.

L'aménagement du cours d'eau Nestos côté grec a suscité bien des polémiques entre les deux pays, la Bulgarie désirant réaliser principalement des ouvrages d'irrigation sur son sol, en amont des aménagements grecs donc, et la Grèce souhaitant un débit minimum garanti à la traversée de la frontière pour alimenter ses centrales hydroélectriques.

Aucun accord n'ayant pu être trouvé, c'est le choix d'une centrale de turbinage-pompage dimensionnée pour un débit d'eau minimum en provenance de Bulgarie qui s'est imposé. 


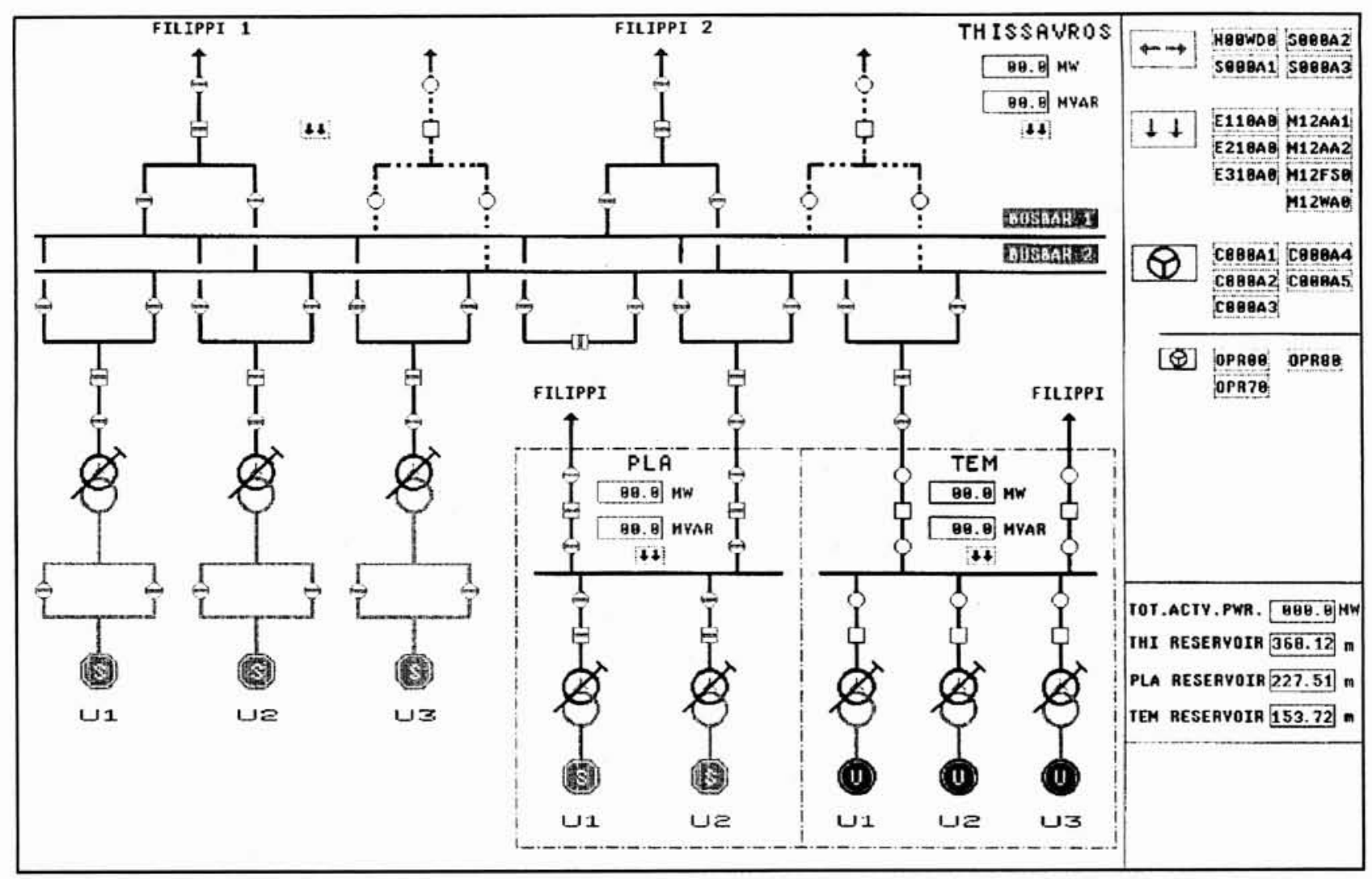

2. Schéma électrique unifilaire de puissance des trois centrales (vue de conduite).

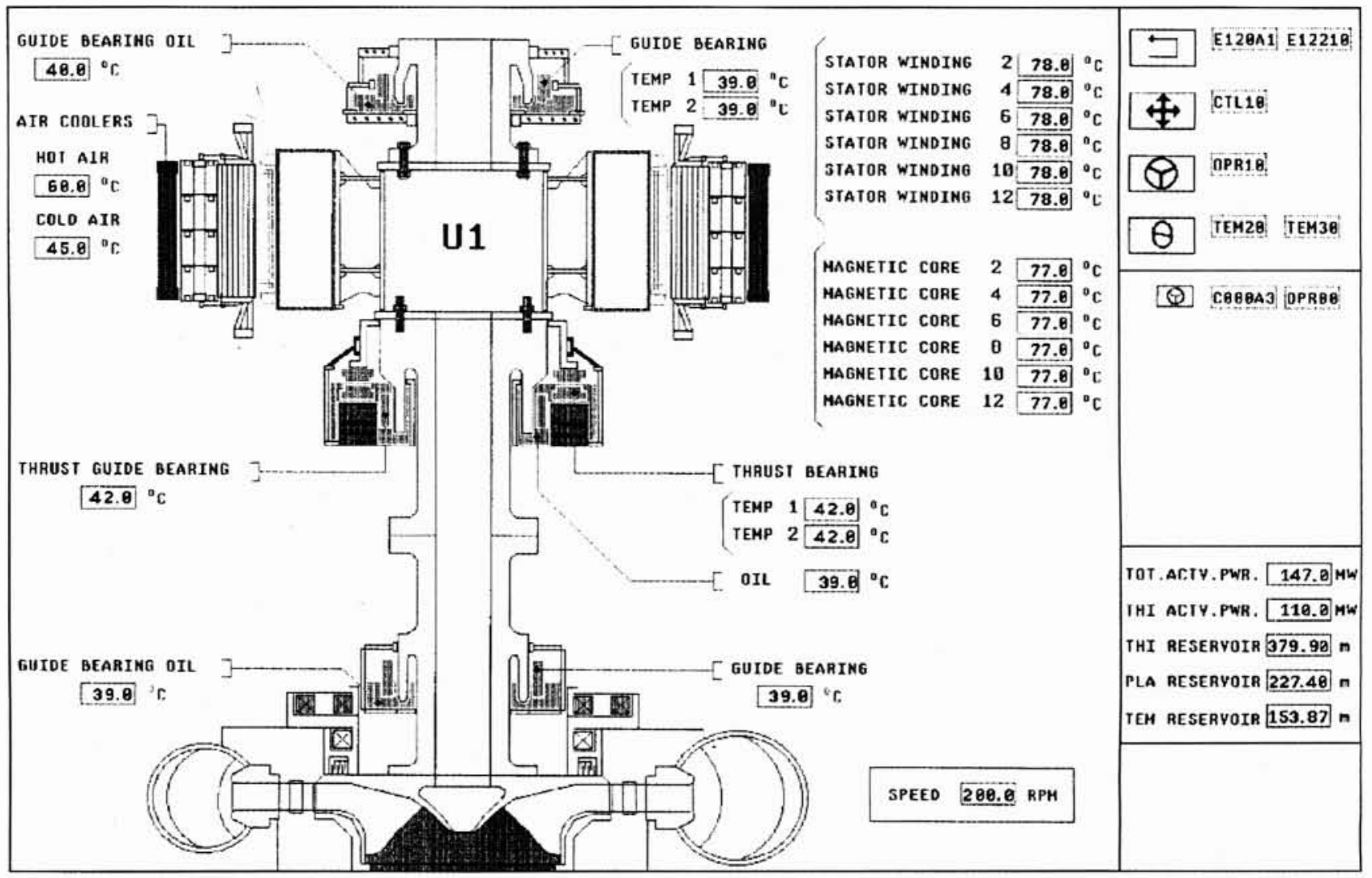

3. Coupe d'un groupe de puissance de Thissavros (vue de conduite). 


\subsection{Les centrales turbine-pompe}

Avant d'examiner les particularités de la centrale de Thissavros, il convient de rappeler les caractéristiques propres à une centrale réversible.

\subsubsection{Intérêt}

Les centrales turbine-pompe ont la rapidité de modulation des centrales hydrauliques et permettent d'ajuster la production d'énergie électrique à la demande des consommateurs. Elles évitent de moduler inutilement la puissance des centrales fournissant l'énergie de base en transférant l'énergie en excès des heures de faible consommation vers les heures de forte consommation. Enfin, elles constituent une réserve d'énergie susceptible d'être utilisée lors d'un retrait inopiné d'une centrale importante du réseau.

\subsubsection{Constitution}

Une installation de turbinage pompage est une centrale hydraulique qui échange l'eau entre un bassin supérieur et un bassin inférieur.

L'eau du bassin inférieur est pompée vers le bassin supérieur pendant les heures creuses, de faible consommation, du réseau électrique.

Elle est ensuite turbinée du bassin supérieur vers le bassin inférieur pendant les heures de pointe de forte consommation.

\subsubsection{Rendement économique}

Le $\mathrm{kWh}$ produit pendant les heures de forte consommation peut se vendre plusieurs fois le prix du $\mathrm{kWh}$ consommé pendant les heures creuses. Ainsi, bien que le rendement énergétique global du pompage turbinage soit inférieur à un, son rendement économique est très supérieur à l'unité !

\subsubsection{Cycles de fonctionnement}

Suivant les capacités des bassins, la puissance installée et les besoins du réseau, plusieurs cycles de fonctionnement peuvent être envisagés :

- Cycle journalier, avec transfert d'énergie des heures creuses de la nuit, vers les heures pleines du jour.

- Cycle hebdomadaire, avec stockage de l'énergie des heures creuses de fin de semaine et de stockage durant les heures de pointe des jours ouvrables.

- Cycle saisonnier, avec stockage estival et déstockage hivernal et bien sûr.

- Cycle mixte, combinant plusieurs des possibilités précédentes.

\subsection{La centrale de Thissavros}

Le bassin supérieur est constitué par le lit de la rivière elle-même en amont du barrage. Le bassin inférieur est constitué par la retenue de la centrale Platanovryssi en aval sur le Nestos.

Tous les cycles de pompage et en particulier les cycles journaliers seront possibles. Quant au turbinage, il devrait surtout être nécessaire tôt le matin, le midi et le soir, c'est-à-dire aux heures de forte demande d'électricité domestique.

La centrale est en caverne ; elle sera connectée au réseau électrique de transport d'énergie via le poste $150 \mathrm{kV}$. C'est aussi de ce poste que partiront en particulier une ligne vers chacune des centrales futures, en aval sur la rivière Nestos, Platanovryssi et Temenos éloignées respectivement de 11 et $15 \mathrm{~km}$ de Thissavros (fig. 2).

A partir des transformateurs principaux situés dans la caverne partent les liaisons en câble $150 \mathrm{kV}$ vers le poste extérieur $150 / 20 \mathrm{kV}$, à côté duquel se trouve le bâtiment d'exploitation et de service. La salle de commande, centre nerveux du projet se trouve dans ce bâtiment.

\subsection{Démarrage en pompe}

Les conditions de démarrage en pompe sont parmi les caractéristiques les plus marquantes des centrales turbinepompe.

Suivant l'alimentation choisie pour l'alternateur-moteur à démarrer en moteur, plusieurs types de démarrage du groupe en pompe sont théoriquement possibles, mais pour Thissavros, seul le démarrage en dos à dos a été retenu.

Dans ce dernier cas, l'alternateur-moteur à démarrer est alimenté par un groupe "lanceur »voisin de la même centrale ou même par un groupe d'une centrale voisine. Le groupe lancé est alimenté par le groupe lanceur, soit au travers du poste HT, soit via le réseau MT. Le groupe lancé est ainsi progressivement amené de l'arrêt à la vitesse nominale. Il peut alors être couplé sur le réseau et libérer le lanceur pour une autre opération. C'est le cas de Thissavros où le démarrage d'un groupe en pompe peut se faire soit par un groupe voisin, soit par un groupe de la centrale de Platanovryssi en aval, via le poste $150 \mathrm{kV}$.

Généralement, dans ce type de centrale, seuls certains groupes sont affectés au démarrage de façon à ne pas compliquer inutilement les automatismes. Parmi les avantages d'une solution de démarrage en dos à dos, on peut noter le peu d'équipements supplémentaires qu'elle nécessite, et la possibilité de démarrer avec la roue de la turbine noyée.

En revanche, ce type de démarrage est relativement long, et impose le fonctionnement d'un groupe turbine en lanceur.

On notera que pour faire fonctionner en pompe les trois groupes de Thissavros, il est nécessaire d'utiliser un des groupes de Platanovryssi pour démarrer le dernier groupe. En effet, il n'est pas prévu d'avoir recours à d'autres types de démarrage tels que l'emploi d'un convertisseur statique de fréquence (CSF) pour Thissavros.

\subsection{Exploitation et contrôle-commande}

Une centrale turbine-pompe doit par définition être disponible à tout moment, pour répondre à la demande d'énergie provenant du dispatching.

Cette haute disponibilité implique un choix soigneux des équipements et une organisation optimale du système de contrôle-commande. Les changements de marche fréquents qui sont l'exploitation normale de ce type de centrales, imposent un nombre élevé de manœuvres aux groupes et aux équipements associés et requièrent donc une 
qualité technique de haut niveau propre à supporter un régime sévère de fonctionnement.

\subsubsection{Comportement à fréquence variable}

Quel que soit le mode de démarrage adopté, certains équipements vont voir leurs performances affectées par une variation de fréquence du courant qui les traverse. Au moment de leur définition, leur comportement particulier devra être pris en compte :

- Transformateur de puissance : prise en compte d'éventuelles surinductions.

- Transformateurs de mesure : saturation affectant la mesure émise.

- Disjoncteur : pouvoir de coupure en basse fréquence. - Relais de protections électriques : adaptation de leur rôle à la phase de démarrage.

Les solutions apportées consistent suivant le type d'équipement soit à imposer des caractéristiques bien précises aux matériels concernés, soit à tenir compte de leur comportement dans l'organisation du contrôle-commande.

\subsubsection{Contôle-commande}

Par rapport à une centrale hydraulique classique, la centrale turbine-pompe présente les particularités d'avoir un nombre très important d'états stables et de transitions associées à gérer.

Le système de contrôle-commande devra donc posséder d'importantes capacités de traitement afin de pouvoir gérer un très grand nombre d'informations.

Fourni et mis en œuvre par Cegelec, il est fondé sur une architecture hiérarchisée de calculateurs Centralog, et sur des automates programmables Controbloc, l'ensemble assurant au client une disponibilité particulièrement élevée.

D'une structure modulaire et totalement redondant, il est organisé en deux niveaux :

- le niveau des unités fonctionnelles équipé d'automates Controbloc associés à chaque groupe, au barrage, au déversoir, au poste $150 \mathrm{kV}$ ainsi qu'aux centrales à l'aval de Platanovryssi et Temenos,

- le niveau de la conduite centralisée équipé du système de conduite Centralog. Ce système comprend des stations graphiques de conduite et de maintenance, des serveurs de données et un système de communication. Toutes ces stations, au standard VME, sont interconnectées sur un réseau doublé de type Ethernet.

Comme pour toute station de turbinage-pompage, sûreté opérationnelle et haute disponibilité sont privilégiées pour répondre en permanence aux besoins du réseau; mais aussi, et c'est une particularité du contrôle-commande de Thissavros, le système calculateur Centralog doit pouvoir se comporter en poste de conduite de rivière et piloter de fait les trois centrales de Thissavros, Platanovryssi et Temenos. Cet aspect est développé ci-après sous le titre «Thissavros, une centrale tête de vallée - Conduite coordonnée de plusieurs aménagements ».

La centrale de Thissavros proprement dite (poste extérieur compris) est exploitée normalement "à distance ", depuis la salle de commande du bâtiment d'exploitation et services, à partir des informations reçues du dispatching.
Deux autres niveaux, locaux, de commande sont possibles pour les groupes, soit manuel depuis les tableaux des groupes, soit depuis la salle de contrôle en caverne.

En salle de commande (bâtiment d'exploitation et services), les principales fonctions réalisées sont (procédé hydraulique et gestion des usines aval mis à part) :

- supervision de toute la centrale,

- démarrage/arrêt de chaque groupe (en pompe ou en turbine),

- réglage de tension,

- réglage de puissance de la centrale par consigne ou par diagramme,

- configuration du réseau.

Toutes ces opérations sont validées et exécutées par le Centralog.

La redondance complète du système permet une disponibilité supérieure à $99,95 \%$.

Toutefois un synoptique mural et un pupitre conventionnel de commande permettent en parallèle à l'opérateur une conduite et une supervision simplifiées de la centrale.

Le Centralog met à la disposition de l'opérateur toutes les informations et tous les événements de l'exploitation sur écran et sur imprimante. La langue utilisée pour les affichages sur écrans et pour les journaux est soit le grec, soit l'anglais, à la demande de l'opérateur.

\section{THISSAVROS, UNE CENTRALE TÊTE DE VALLÉE - CONDUITE COOR- DONNÉE DE PLUSIEURS AMÉNAGE- MENTS}

\subsection{Gestion de la puissance globale}

Le but de la conduite automatique des aménagements est de permettre l'introduction d'un plan de puissance globale pour l'ensemble des trois usines en cascade et sa réalisation automatique et optimisée sur les trois usines (fig. 4).

Ce plan de puissance programmé pour 1 ou 2 jours est introduit depuis l'une ou l'autre des deux stations de travail de la salle de commande et est visualisé sous forme graphique (fig. 5). La consigne globale peut également être télétransmise au système de conduite depuis le dispatching national d'Athènes.

L'exécution de ce plan de puissance est à la charge du logiciel d'application du système calculateur Centralog qui doit résoudre un problème d'optimisation non seulement à chaque réception d'un nouveau plan de puissance, mais également de manière permanente en fonction des contraintes imprévues telles que les déclenchements ou blocages des machines, ou les modifications des apports hydrauliques naturels.

Ce logiciel de conduite appliqué a été personnalisé et assemblé à partir d'une bibliothèque de modules standard HYDROSET. Chaque module, écrit en langage $C$, a été développé et validé suivant une méthodologie rigoureuse dans un environnement atelier logiciel et mis au point et amélioré sur de nombreux sites hydrauliques. En particulier, les modules concernant la conduite de vallées et la gestion de plusieurs usines en cascade ont été développés à partir de l'expérience acquise par Cegelec au cours des projets français des vallées du Rhône, de la Durance et du Rhin. 


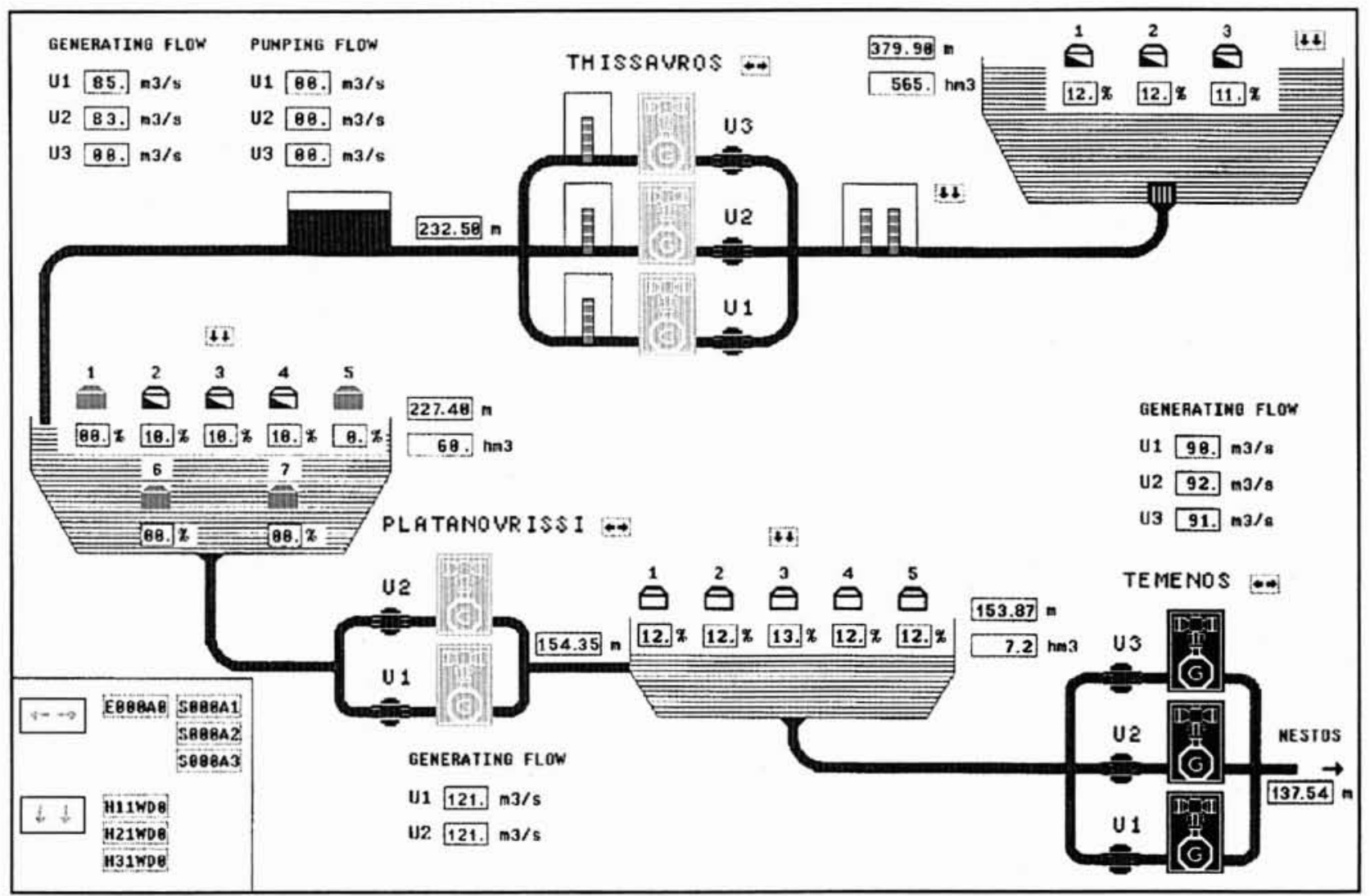

4. Schéma hydraulique de l'ensemble des trois centrales (vue de conduite).

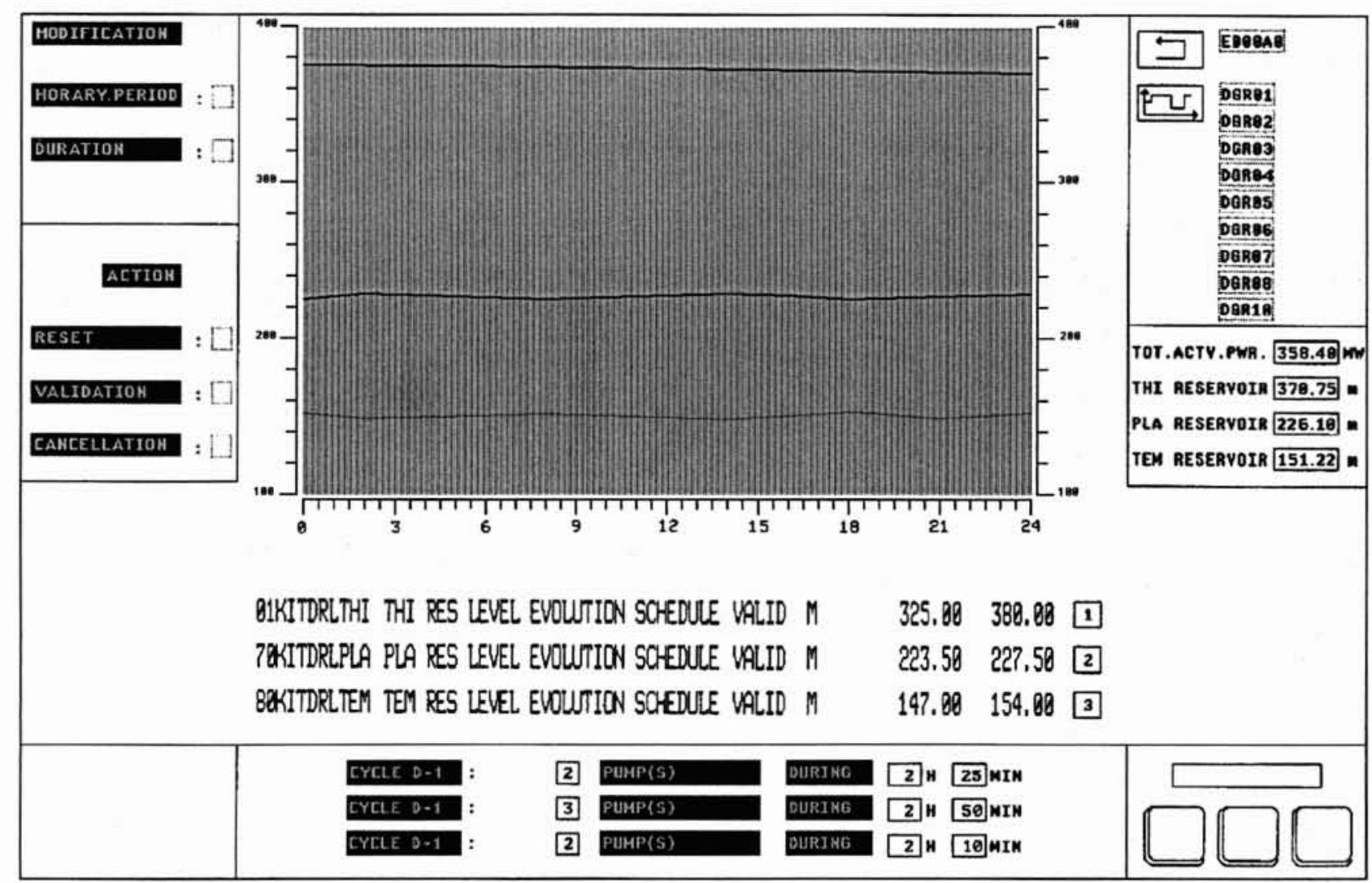

5. Diagramme de modulation type (vue de conduite). 
Ici, la vallée comprend une usine de tête (Thissavros) pouvant turbiner ou pomper de manière très irrégulière, avec un réservoir de stockage de type annuel (565 millions de mètres cubes), une usine intermédiaire (Platanovryssi) avec un réservoir moyen ( 60 millions de mètres cubes) et une usine de queue (Temenos) avec une capacité de stockage faible ( 7 millions de mètres cubes) et donc contrainte à un fonctionnement «au fil de l'eau ».

Cette configuration a conduit à réaliser une optimisation à trois niveaux :

- Le premier niveau est un guide opérateur de prévision à long terme. Il permet à l'opérateur, grâce à un modèle intégré des trois usines et bassins, de consulter à tout moment les évolutions prévisionnelles des niveaux dans les deux premiers bassins compte tenu du plan de puissance et de prévisions de disponibilité des organes et des débits entrants naturels prévus dans l'année.

- Le deuxième niveau entre en jeu à chaque introduction d'un plan journalier de puissance global. Il permet le contrôle de la faisabilité du plan global, sa répartition sur les trois usines selon une stratégie fine et l'affichage graphique de l'évolution prévisionnelle des niveaux dans les trois bassins à l'échelle de la journée. La stratégie de répartition de la production entre les trois usines repose sur le meilleur compromis entre les besoins de minimiser la consommation globale d'eau et le respect des nombreuses contraintes d'exploitation (minimiser les arrêts et démarrages des groupes, respecter les contraintes d'irrigation à l'aval de l'aménagement, les prévisions de consignation des groupes, les contraintes de niveaux et enfin permettre les cycles de pompage eux aussi programmés à l'avance). Cette gestion prévisionnelle de la puissance est également réalisée périodiquement afin de prendre en compte au plus tôt toute nouvelle contrainte.

- Le troisième niveau est l'optimisation en temps réel de la conduite : chaque usine doit respecter son propre plan de production. Les groupes sont pilotés de manière à optimiser le rendement de l'usine et respecter les contraintes déjà citées.

Dans chaque bassin, le système contrôle le respect de l'évolution prévisionnelle du niveau. D'autre part, lorsque les niveaux se rapprochent des limites autorisées, un régulateur intervient et agit sur les vannes déversoirs.

\subsection{Gestion des cycles de pompage}

Les cycles de pompage sont préprogrammés comme des programmes de puissance négative. Le suivi de ces programmes et les démarrages en pompe des groupes de Thissavros sont réalisés automatiquement. A chaque démarrage en pompe, en mode dos à dos, le calculateur joue le rôle de chef d'orchestre en choisissant le groupe à démarrer, en désignant le groupe lanceur et en initialisant les séquences réalisées par chaque automate de groupe.

\subsection{Régulation de tension et de fréquence}

Outre ces fonctions de conduite hydraulique, le calculateur Centralog effectue également la régulation de la tension des jeux de barres du poste $150 \mathrm{kV}$ en agissant sur l'excitation des générateurs de Thissavros et de Platanovryssi. Enfin, il réalise un réglage secondaire de la fréquence en ajoutant une action correctrice sur les régleurs de chaque turbine afin de maintenir constante la fréquence du poste.

\subsection{Information de l'opérateur}

L'interface homme-machine constituée de deux stations opérateurs graphiques à deux écrans chacune a été particulièrement étudiée pour permettre à l'opérateur de lancer différents scénarios avec différents plans de production et simulation d'indisponibilité d'organes avant de valider son choix. D'autre part, tout phénomène imprévu lors de la réalisation du programme est signalé et une solution optimale dégradée est adoptée.

\section{IV $\square$ CONCLUSION}

La centrale hydroélectrique de Thissavros en Grèce est une affaire remarquable. Située dans le nord-est de la Grèce entre la ville de Drassa et la frontière bulgare, à une distance d'environ $200 \mathrm{~km}$ à l'est de Thessalonique, Thissavros, avec ses trois groupes réversibles de 140 MVA, sera la centrale de ce type la plus importante de Grèce.

Avec une augmentation de $13 \%$ de la puissance hydraulique installée, elle renforcera le réseau grec surtout localement. En effet, les régions de Thrace et de Macédoine connaissent une forte croissance de leur demande en électricité en raison du programme actuel d'industrialisation destiné à enrayer l'exode rural. La centrale est en cours de réalisation par un groupement international avec une forte participation française Cegelec. C'est une centrale de turbinage-pompage, capable de transférer de l'énergie des heures de faible consommation vers les heures de forte consommation. Enfin, elle constitue la tête d'un ensemble de trois aménagements hydroélectriques sur un même fleuve et aura à ce titre à assurer le fonctionnement cohérent de cet ensemble.

Cegelec, grâce à ses capacités d'études et à son expérience dans ce domaine, a relevé ces trois défis avec une solution globale, cohérente et ouverte. 